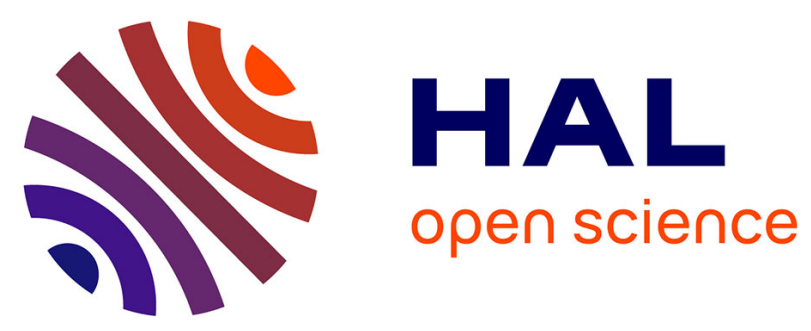

\title{
Partial Human Data in Design of Human-Like Walking Control in Humanoid Robotics
}

\author{
David Galdeano, Vincent Bonnet, Moussab Bennehar, Philippe Fraisse, \\ Ahmed Chemori
}

\section{- To cite this version:}

David Galdeano, Vincent Bonnet, Moussab Bennehar, Philippe Fraisse, Ahmed Chemori. Partial Human Data in Design of Human-Like Walking Control in Humanoid Robotics. 10th IFAC Symposium on Robot Control, Syroco 2012, Sep 2012, Dubrovnik, Croatia. pp.485-490, 10.3182/20120905-3-HR2030.00187 . hal-00717243

\section{HAL Id: hal-00717243 \\ https://hal.science/hal-00717243}

Submitted on 12 Jul 2012

HAL is a multi-disciplinary open access archive for the deposit and dissemination of scientific research documents, whether they are published or not. The documents may come from teaching and research institutions in France or abroad, or from public or private research centers.
L'archive ouverte pluridisciplinaire HAL, est destinée au dépôt et à la diffusion de documents scientifiques de niveau recherche, publiés ou non, émanant des établissements d'enseignement et de recherche français ou étrangers, des laboratoires publics ou privés. 


\title{
Partial Human Data in Design of Human-Like Walking Control in Humanoid Robotics
}

\author{
David Galdeano* Vincent Bonnet ${ }^{\dagger}$ Moussâb Bennehar* \\ Philippe Fraisse* Ahmed Chemori* \\ * LIRMM, Université Montpellier 2, LIRMM - UMR 5506 - CC 477, \\ 161 rue Ada, 34095 Montpellier Cedex 5, France \\ (e-mail:galdeano(Bennehar,chemori,fraisse)@lirmm.fr). \\ $\dagger$ LABLAB, Department of Human Movement and Sports Sciences, \\ University of Rome "Foro Italico", Piazza Lauro de Bosis 6, 00194 \\ Rome, Italy. (e-mail:v.bonnet@uniroma4.it).
}

\begin{abstract}
In this paper a human-based control strategy is proposed for walking of humanoid robots. Its basic idea lies in the control of the CoM as well as the relative position of robot's feet. Through the proposed study, it is shown that this reduced set of data allows to fully describe and reproduce a whole body human-like walking. In addition of reducing the number of controlled variables, the proposed control strategy has the advantage of not requiring a complete walking cycle decomposition due to its continuous character. Simulations results are presented to show the effectiveness of the proposed control scheme.
\end{abstract}

Keywords: Humanoid robotics, Human motion capture, Walking control.

\section{INTRODUCTION}

In humanoid robotics, the challenge to perform stable dynamic walking under inherent difficulties in controlling such highly non-linear redundant structures is still an open problem. In the literature, many control approaches using high level pattern generators have been proposed. One of the problems in using such methodology is to determine the proper variables and constraints able to describe efficiently a stable walking pattern.

To solve this problematic, many researches have been conducted focusing on lower-limbs motion. However, only few studies on generating human-like whole-body motion have been conducted. Indeed, due to their anthropomorphic structure, humanoid robots often present dynamic similarities with humans. Hence, modern pattern generators rely on captured human data obtained from motion capture systems in order to realize human-like tasks such as balancing Montecillo-Puente et al. (2010), walking Kim et al. (2009), or dancing Montecillo-Puente et al. (2010), Kim et al. (2009). However, despite evident similarities, the human locomotor system is much more complex than the humanoid robots structures. Therefore, a direct mapping from human captured motion to humanoid robot will fail in most cases and often lead to the fall of the robot. This implies that data need to be adapted to take into account the specific structures of humanoid robots.

In the literature, several approaches have been proposed to imitate human motion; they can be classified into two classes: on-line and off-line pattern generators.

Within the first class, a humanoid-normalized model Montecillo-Puente et al. (2010) based on a real-time task- based prioritized inverse kinematics solver is used to transfer captured motion to the humanoid robot. Two scenarios were experienced to validate this method: a dancing task and a single foot balancing motion. However, the execution of all tasks is not guaranteed since the Zero Moment Point (ZMP) and joints limits constraints could not be satisfied.

In Yamane and Hodgins (2010), a balance controller, using a linear quadratic regulator associated to an inverted pendulum model, and a joint space controller were used to generate joint torques allowing the robot to track the estimated human trajectories. A captured Tai-chi motion was experienced to assess the performance of this joint controller. However, no walking motion has been experienced.

A neural network approach has been proposed by Schaal and his coworkers $(1999,2003)$, where human data issued from motion capture were used to feed a learning system to produce accurate movement primitives. The drawbacks of such a method are the required learning time and the low accuracy. Hence, this method cannot be really applied for highly dynamic tasks such as walking.

Off-line methods are generally more efficient than realtime ones since the captured data can be processed separately and then transferred to the humanoid robot. Many approaches propose to process human walking and extract different gait parameters such as step length or step duration. Using these global parameters, joint trajectories can be determined and adapted to specific humanoid structures.

Different research teams such as Harada et al. (2009), Miura et al. (2009) successfully reproduced the hu- 
man lower-limbs kinematics during walking on humanoid robots. It is worth to note that the dynamical balance and the upper-limbs motions were not taken into account in the above presented schemes. To overcome balance limitation, a human whole-body motion using the ZMP obtained from a simplified human model was recently proposed Kim et al. (2009). Although, no walking has been experimented with this method, a complex dancing motion was accurately imitated in simulation by the humanoid.

Another method was proposed in Nakaoka et al. (2003) using inverse kinematics for upper-limbs and motion primitive for lower-limbs. In this method, upper-limbs and lower-limbs trajectories are generated separately. The combination of both trajectories is time consuming as well as sometimes unstable.

In Ramos et al. (2011), a method to imitate human motion using stack of tasks was proposed, where the robot is expected to follow the joint trajectories extracted from human motion capture. The authors add some specific arbitrary tasks (such as: knee oscillation, hand motion, foot sliding) to adjust the humanoid robot dynamics, enhancing therefore the human motion imitation.

An identification-based method for modeling human walking was proposed in Suleiman et al. (2006). The human body was decomposed into five open kinematic chains and considered as a black-box system. The only data needed to obtain the other limbs' trajectories was the pelvis's trajectory. Their method was then validated through simulation using artificial and human captured pelvis trajectories.

This paper presents a whole-body control strategy. We use center of mass and relative feet position issued from human motion capture as desired trajectory and, using Nakamura et al. (1987) formalism, we can track these two quantities. With this strategy, we can create a continuous whole body human-like walking.

This paper is organized as follows. In section 2 , an overview of the motion capture system is introduced, where two main points are discussed: the proposed human model and the human data collection. Section 3 is devoted to the proposed control scheme, where its basic principle is presented and discussed. Simulation results are presented in section 4, including two main parts; in the first one a description of the developed simulator is introduced, whereas the second one deals with a presentation and discussion of the obtained results. The paper ends with some concluding remarks and future work.

\section{DESCRIPTION OF MOTION CAPTURE SYSTEM}

\subsection{Human model}

In this study, the human subject (cf. Fig.1) is considered as a multibody rigid system with 22 degrees of freedom (dof). These dof are distributed on the different articulations of the human model as illustrated in Fig. 1. All degrees of freedom are pure rotations.

Each leg is composed of six dof, three dof at the hip, one dof at the knee and two dof at the ankle. The trunk and the neck are composed of 2 dof on the frontal plane each.

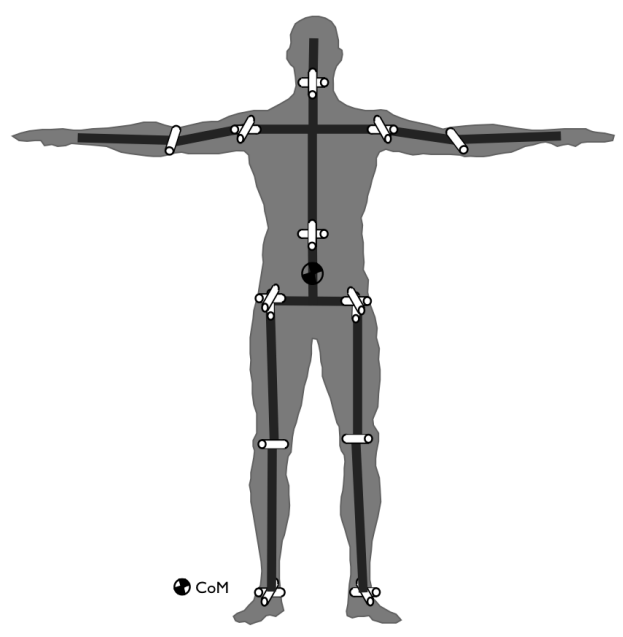

Fig. 1. Illustration of the kinematic model used to represent the human locomotor system (degrees of freedom in articulations).

Both arms are composed of 3 dof, 2 dof at the shoulder and one dof at the elbow.

The limbs' lengths are measured on the subject and used in the proposed human model. The mass, center of mass and inertia of each body are computed using the anthropomorphic table given in De Leva (1996).

\subsection{Human data-collection}

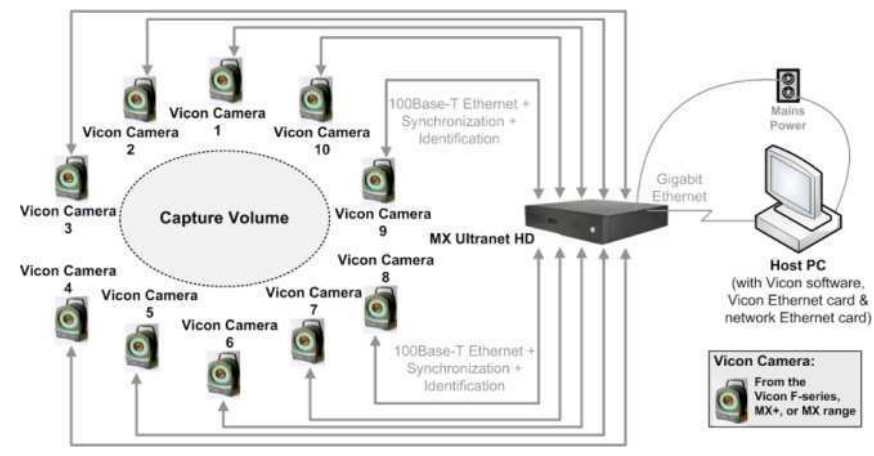

Fig. 2. A typical motion capture setup.

One volunteer (age: 35 years, height: $1.71 \mathrm{~m}$, weight: $72 \mathrm{~kg}$ ) participated to the data-collection experiments after signing an informed consent form. Anthropometric data (limbs' lengths) were initially collected in upright position and used in the previously mentioned human model (cf. section 2.1). The volunteer was asked to initiate gait at his preferred speed, starting from natural upright position, to perform one stride. The choice of the first swing leg was let free to the subject. A nine-camera system (MX, VICON) (cf. Fig.2) was used to record the 3D trajectories of the 35 reflective markers (cf. Fig. 3-(a)) located on anatomical landmarks specified in a commonly-used whole body template model, i.e. Vicon PeakßPlug-In-Gait.

The recorded reflective markers were used to lead the kinematic model described and illustrated in section 2.1 in order to estimate joint kinematics. Thanks to these joint trajectories the CoM location and feet trajectories were estimated in the global reference frame (cf. 3-(c)). The 


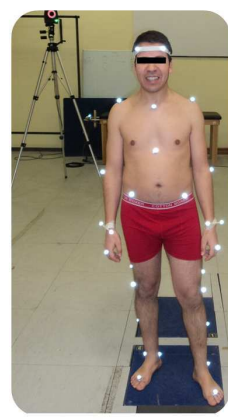

(a)

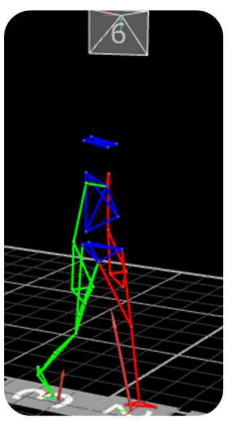

(b)

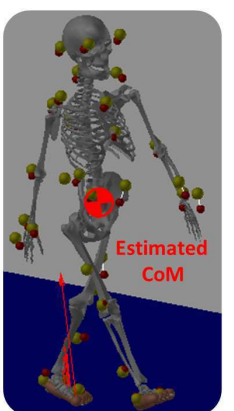

(c)
Fig. 3. Basic principle of human kinematic data acquisition. A whole body template of 35 reflective markers (a) was used to track the motion of each limb (b). The corresponding markers were used to lead a specified kinematic model (c) allowing the estimate of joint and feet trajectories, and Center of $\operatorname{Mass}(\mathrm{CoM})$.

joint kinematic estimate was performed using a commercial biomechanical software (LIFEmod Brg). The reliability and accuracy of Plug-In-Gait template for the CoM estimate during walking has been demonstrated in human movement science for healthy people in Gutierrez-Farewik et al. (2006).

\section{PROPOSED CONTROL SCHEME}

As mentioned in the introduction, the challenge of using human trajectories to lead the design of a human-like pattern generator for humanoid robots is not yet adressed. Therefore, a reduction of the used data set would be necessary to keep the most valuable information. Furthermore, this can help for a better understanding of human walking and a smarter implementation of human-like walking on humanoid robots.

To this purpose, we propose to select only the feet and CoM positions to describe the main features of the human walking. In order to achieve the tracking of these two quantities, the redundancy of humanoid robot is considered through the task-priority formalism as described in Nakamura et al. (1987).

The real human CoM $\left(C o M_{d}\right)$ and relative feet positions $\left(P_{r d}\right)$ obtained from human data collection (described in section 2.2) are used as reference trajectories in the proposed control approach illustrated in Fig. 4.

The starting point of our first task is based on the tracking error $\varepsilon_{r}=P_{r d}-P_{r}$ between the desired feet spacing $P_{r d}$ extracted from motion capture and the estimated feet spacing $P_{r}=P_{r f}-P_{l f}$, where $P_{r f}=\left[\begin{array}{lll}x_{r f} & y_{r f} & z_{r f}\end{array}\right]^{T}$, $P_{l f}=\left[\begin{array}{lll}x_{l f} & y_{l f} & z_{l f}\end{array}\right]^{T}$ are the positions of the right and left foot (respectively) obtained using a forward kinematic model $\left(F K M_{r}\right)$.

The Jacobian of this task is then defined as follows:

$$
\varepsilon_{r}=J_{r} \varepsilon_{q}
$$

where $\varepsilon_{q} \in \mathbb{R}^{22 \times 1}$ represents the vector of articular position errors of the robot, $J_{r} \in \mathbb{R}^{3 \times 22}$ is the Jacobian matrix of the relative translation between the feet.
The angular velocity can be computed by inversing equation (1) as follows:

$$
\varepsilon_{q}=J_{r}^{+} \varepsilon_{r}+\left(\mathbb{I}-J_{r}^{+} J_{r}\right) Y
$$

where $J_{r}^{+}$is the pseudo-inverse of $J_{r},\left(\mathbb{I}-J_{r}^{+} J_{r}\right)$ is the null-space projector of this Jacobian and $Y$ is an arbitrary vector. This equation allows, using $Y$, to achieve some other task without disturbing the primary task.

Our second task is the tracking error of the CoM expressed by $\varepsilon_{C o M}=C o M_{d}-C o M$ where $C o M_{d}=$ $\left[\begin{array}{lll}x_{C o M_{d}} & y_{C o M_{d}} & z_{C o M_{d}}\end{array}\right]^{T}$ is the desired trajectory of the center of mass extracted from motion capture and $C o M=$ $\left[\begin{array}{lll}x_{C o M} & y_{C o M} & z_{C o M}\end{array}\right]^{T}$ is the one obtained from the forward kinematic model of center of mass position $\left(F K M_{C o M}\right)$.

$$
\varepsilon_{C o M}=J_{C o M} \varepsilon_{q}
$$

where $J_{C o M} \in \mathbb{R}^{3 \times 22}$ is the CoM Jacobian matrix.

Replacing (2) in (3) gives the expression of $\varepsilon_{C o M}$ as follows:

$$
J_{C o M} \varepsilon_{q}=\varepsilon_{C o M}=J_{C o M} J_{r}^{+} \varepsilon_{r}+J_{C o M}\left(\mathbb{I}-J_{r}^{+} J_{r}\right) Y
$$

$\tilde{J}_{C o M}$ is defined as a projection in the null space of $J_{r}$ Jacobian of $J_{C o M}$ Jacobian, that is:

$$
\tilde{J}_{C o M}=J_{C o M}\left(\mathbb{I}-J_{r}^{+} J_{r}\right)
$$

Equation (5) replaced in (4) gives:

$$
\tilde{J}_{C o M}^{+}\left(\varepsilon_{C o M}-J_{C o M} J_{r}^{+} \varepsilon_{r}\right)=Y
$$

Injecting equation (6) in equation (2) gives the following final formulation:

$$
\varepsilon_{q}=J_{r}^{+} \varepsilon_{r}+\left(\mathbb{I}-J_{r}^{+} J_{r}\right) \tilde{J}_{C o M}^{+}\left(\varepsilon_{C o M}-J_{C o M} J_{r}^{+} \varepsilon_{r}\right)
$$

With this equation, the part of the second task which is realized by the first task is taken into account.

As the task-priority formalism is used, it is worth to note that the first task has the higher priority, which means that the relative feet position tracking has a higher priority to the CoM position tracking. An important feature is that in case of conflict between tasks, the one with higher priority can still be fully accomplished, while the one with lower priority can be partially accomplished. Since the human model is highly redundant and disposes of many dof, both tasks should be easily achieved.

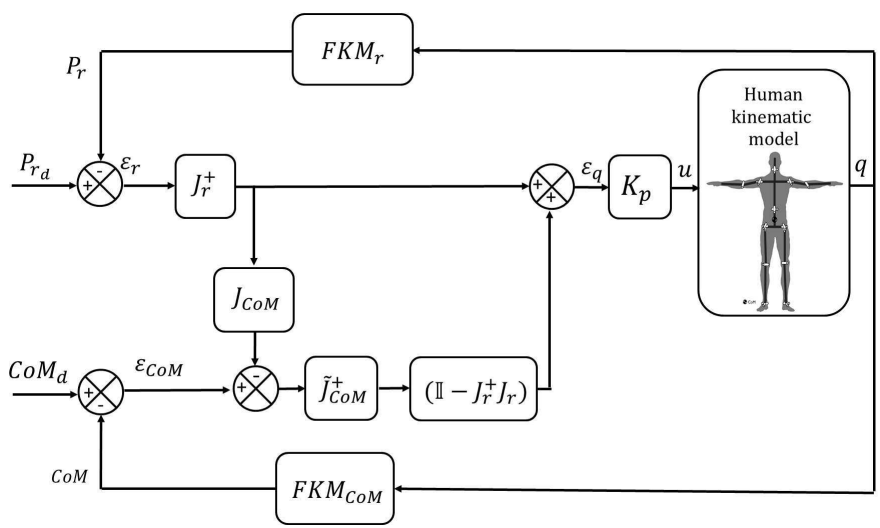

Fig. 4. Block diagram of the proposed control scheme.

The proposed control scheme is summarized in the block diagram of Fig. 4 where $K_{p} \in \mathbb{R}^{22 \times 1}$ is a unitary gain, 
$u \in \mathbb{R}^{22 \times 1}$ is the control input of the human kinematic model, with $u=K_{p} \varepsilon_{q}$ and $q \in \mathbb{R}^{22 \times 1}$ represents the vector of joint positions of the robot.

The human kinematic model is composed of integrators with respect to articular limits.

\section{SIMULATION RESULTS}

\subsection{Developed simulator}

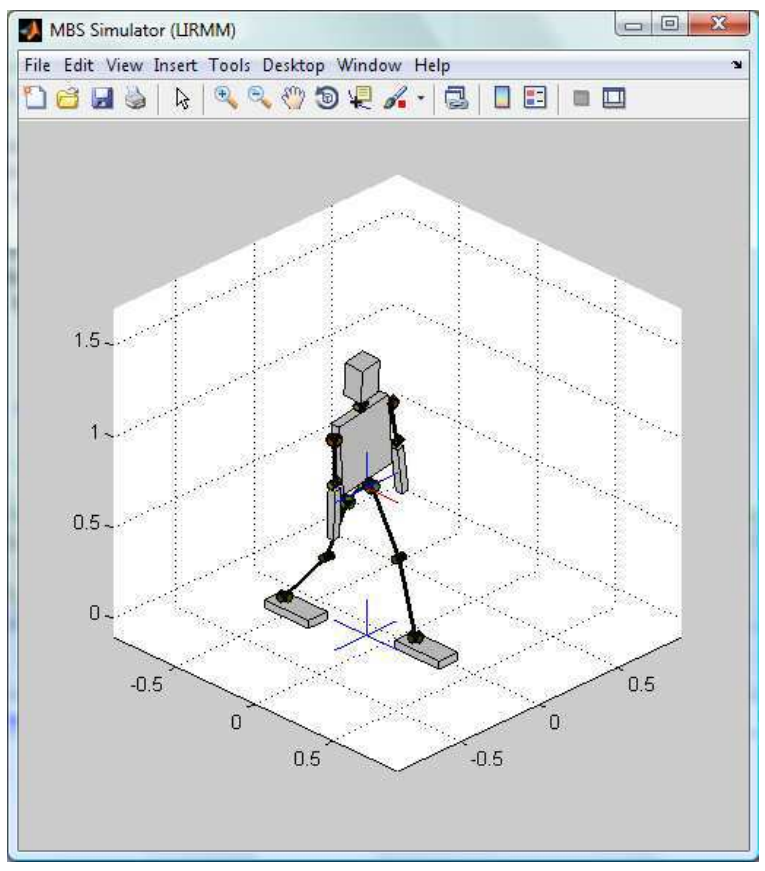

Fig. 5. Graphical interface of the developed simulator.

To illustrate and validate the concepts proposed within this study, a simulator for humanoid robots was developed using Matlab ${ }^{1}$ software. This simulator includes kinematic and dynamic models of different humanoid robots, such as the biped robot SHERPA (a two-leg biped walking robot with 18 dof), HRP2 (a humanoid robot with 30 dof), a generic humanoid model inspired from human (with 20 dof, for whole body motion control), and the human model used in this study (with 22 dof, for human-databased control). To complete the dynamics of the robot with that of its environment, a contact foot/ground model based on the penalty concept has been implemented and integrated in the simulator. The graphical user interface of the developed simulator is shown in Fig. 5. Besides the graphical visualization of the robot, the simulator enables to show in real-time the obtained motions illustrated on a humanoid robot, as well as the evolution of the 3D position of the COM and its projection on the polygon of support. The simulator incorporates the control scheme proposed within this study. However, only human model kinematics is used in simulation of the proposed control strategy. For reasons of illustration and presentation of the obtained results, the simulator allows the user to plot the evolution of all the simulation curves (such as articular trajectories, COM, ZMP, contact forces, etc) and save them in text or images files.

\footnotetext{
1 Matlab is a registered trademark of The Mathworks, Inc.
}

\subsection{Obtained results}

As previously mentioned, the kinematic model of the humanoid robot involves severe simplification with the human locomotor system. The complexity of human model with respect to the humanoid one imposes a need to adapt the human data to the humanoid robot, since a direct mapping will not be efficient. To do so, we propose to adjust the vertical position of CoM to avoid singularities during the walking phase. The modification is done by lowering the vertical position of CoM trajectory up to canceling the tracking errors $\varepsilon_{r}$ and $\varepsilon_{C o M}$.

Once this adaption was validated, we have developed and implemented the control scheme (cf. Fig. 4) in the simulator (presented in the previous section) including the reference signals obtained from motion capture (relative position of feet and CoM location). Three walking steps were considered in this study. We propose in this section to show and analyze two different strategies. The first strategy is without adaptation of vertical position of the CoM. Whereas, the second one takes into account the difference between the human subject and the humanoid model by adjusting the vertical position of CoM to avoid kinematics singularities in computation of the control scheme.

The obtained results of the first strategy are shown on Fig. 6 where we can observe that both arms are raised to satisfy the CoM task. This is due to the difference between human and humanoid models as it has previously been underlined. Some dof are highly solicited such as the ones of the trunk, furthermore high angular velocities during short phases can be observed (cf. for instance consecutive positions of Fig. 6 (e)-(f)). Legs are often straight, this means that the robot is in a singular configuration. Consequently, this strategy cannot be used to generate a human-like walking.

The simulation results of the second strategy are displayed in Fig. 7, a walking pattern is carried out with a correction of the CoM trajectory. We clearly observe that the arms remain along the body. The trunk orientation still has some quick rotation phases. Knees are bended, which means that the robot avoids singularity during the walking phase. This approach is better and closer to human-like than the previous one since it gives a more natural walking.

The obtained joints' trajectories for the right leg in the sagittal plane are shown in Fig. 8. In this second study, it is worth to note that the orientation of the feet is not taken into account (only feet relative position is considered) due to the non consideration of the lift off and landing phases, consequently, a comparison with human data will not be relevant.

The pelvis position in Cartesian space is very close to the human one as displayed in Fig. 9. The simulated pelvis position is lower than the pelvis position from human capture. This is due to the reference CoM adjustment.

\section{CONCLUSION AND FUTURE WORK}

In this paper a control scheme for humanoid walking robot was proposed, based on two tasks controlling both the relative motion of feet and the trajectory of CoM coming from human data. One of the main advantages of this 


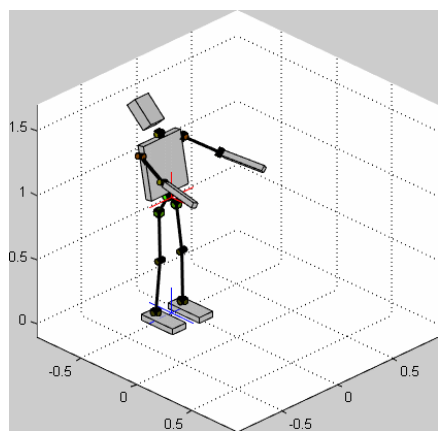

(a) $\mathrm{t}=0.3 \mathrm{~s}$

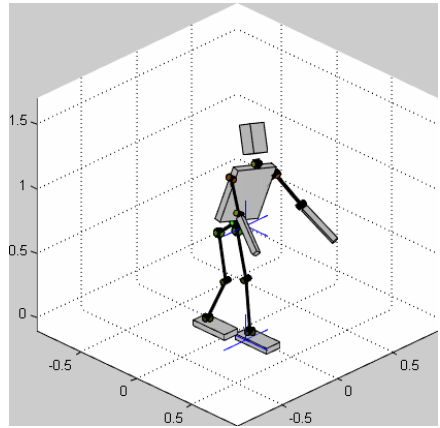

(e) $\mathrm{t}=1.5 \mathrm{~s}$

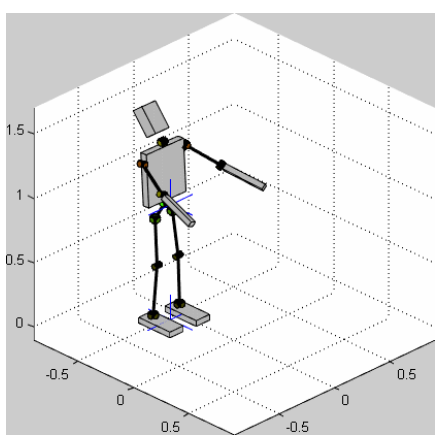

(b) $\mathrm{t}=0.6 \mathrm{~s}$

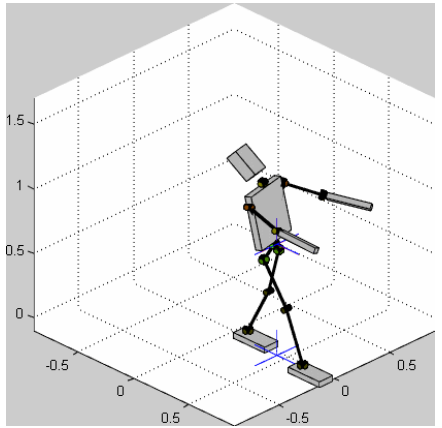

(f) $\mathrm{t}=1.8 \mathrm{~s}$

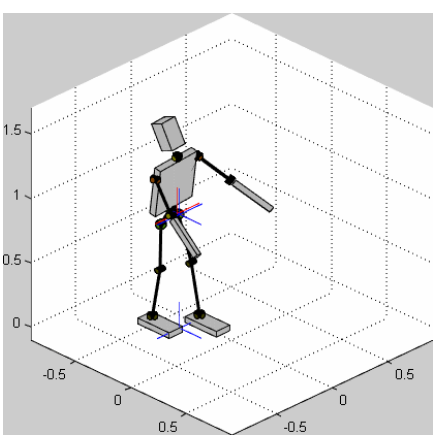

(c) $\mathrm{t}=0.9 \mathrm{~s}$

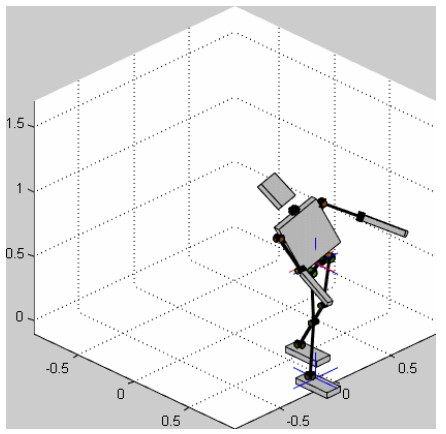

(g) $\mathrm{t}=2.1 \mathrm{~s}$

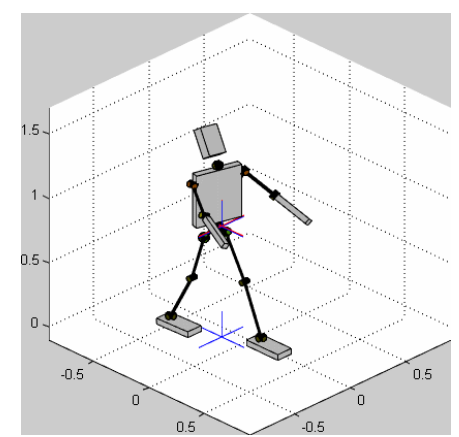

(d) $\mathrm{t}=1.2 \mathrm{~s}$

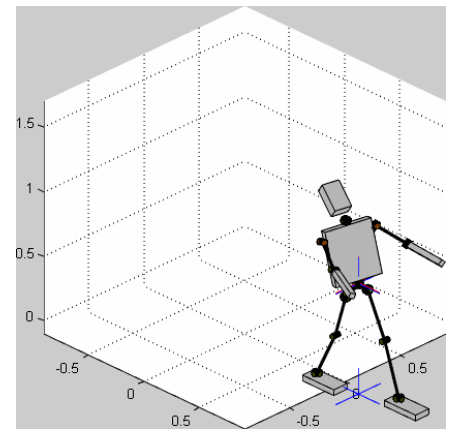

(h) $\mathrm{t}=2.4 \mathrm{~s}$

Fig. 6. Obtained simulation results: First strategy (without CoM adaptation).

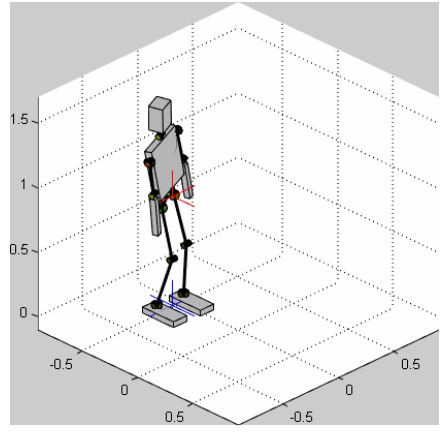

(a) $\mathrm{t}=0.3 \mathrm{~s}$

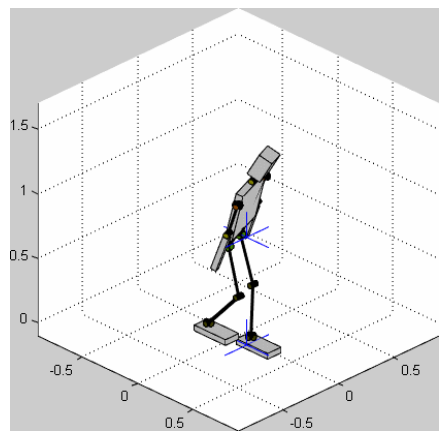

(e) $\mathrm{t}=1.5 \mathrm{~s}$

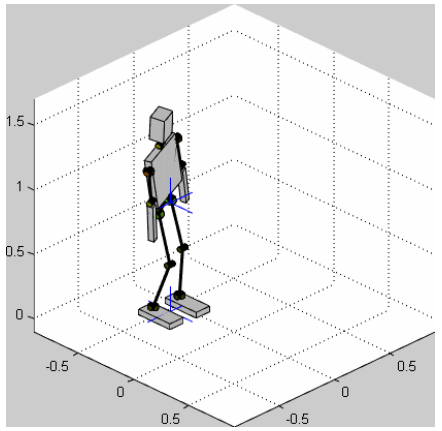

(b) $\mathrm{t}=0.6 \mathrm{~s}$

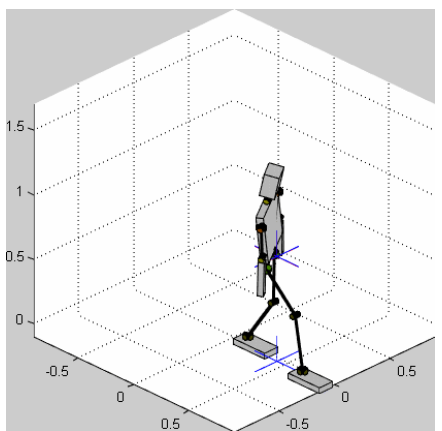

(f) $\mathrm{t}=1.8 \mathrm{~s}$

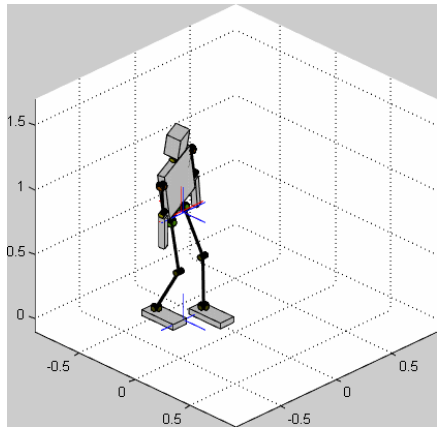

(c) $\mathrm{t}=0.9 \mathrm{~s}$

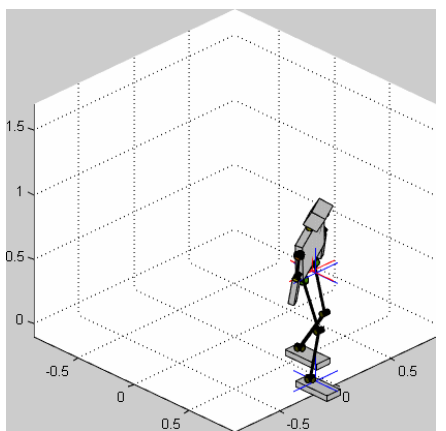

(g) $\mathrm{t}=2.1 \mathrm{~s}$

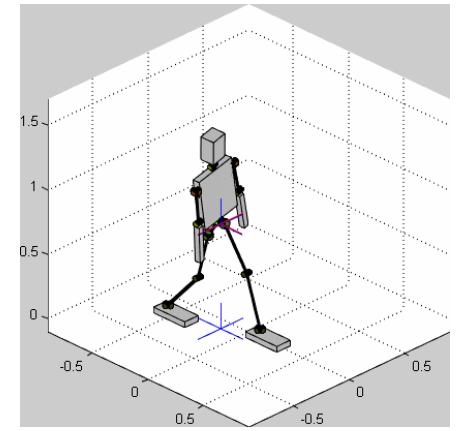

(d) $\mathrm{t}=1.2 \mathrm{~s}$

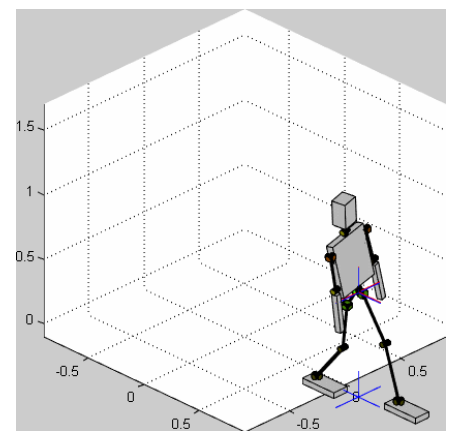

(h) $\mathrm{t}=2.4 \mathrm{~s}$

Fig. 7. Obtained simulation results: Second strategy (with CoM adaptation).

approach is to provide a continuous control framework as well as human-like walking without imitation such as direct mapping in the joint space for instance.
We showed, according to the control scheme that we are able to control the whole-body motion for walking phases with only CoM and relative motion of feet. This is a new approach and the first results are very promising and challenging. However, we had to adapt the vertical position 


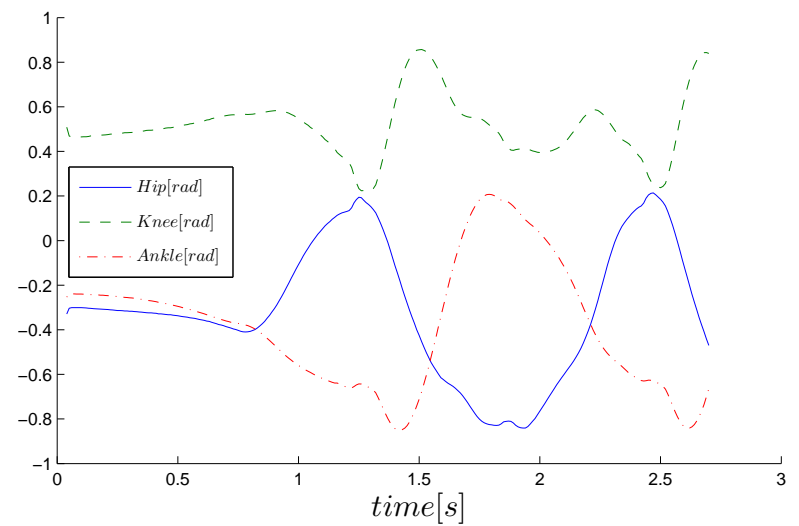

Fig. 8. Right leg joint angular trajectories in the sagittal plane.

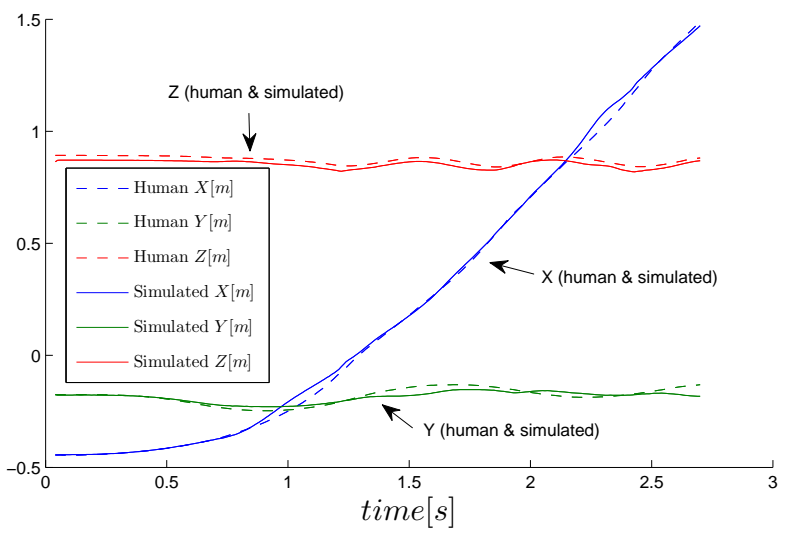

Fig. 9. Cartesian pelvis position, where $X, Y$ and $Z$ represent respectively the antero-posterior, mediolateral and vertical axis.

of CoM issued from human walking to avoid singularities and abnormal postures during walking.

In future work, we aim at improving this approach by considering the contact forces with the ground (ZMP) while adapting automatically the vertical CoM location as a constraint in the controller.

In human, it has been shown that the natural motion of the arms results from a passive control in Collins et al. (2009). The term passive here refers to a spring-damping system at each joint of the arm. This observation underlines that the motion of the whole body propagates to the arms to generate a passive swing. It has also been shown that the natural coordination between the arms and the legs tends to minimize the moment around the vertical axis of the ground reaction forces. We believe that the use of a dynamic model, in future extensions of the present work, should allow us to observe the motion of the arms.

\section{ACKNOWLEDGEMENTS}

This research was supported by the French National Research Agency, within the project R2A2 (ANR-09-SEGI011) and in part by the Egide Galileo project (PHC $\left.\mathrm{n}^{\circ} 26078 \mathrm{TE}\right)$.

\section{REFERENCES}

Collins, S., Adamczyk, P., and Kuo, A. (2009). Dynamic arm swinging in human walking. Proceedings of the Royal Society B: Biological Sciences, 276(1673), 36793688.

De Leva, P. (1996). Adjustments to zatsiorsky-seluyanov's segment inertia parameters. Journal of Biomechanics, 29(9), 1223-1230.

Gutierrez-Farewik, E., Bartonek, A., and Saraste, H. (2006). Comparison and evaluation of two common methods to measure center of mass displacement in three dimensions during gait. Human Movement Science, 25(2), 238-256.

Harada, K., Miura, K., Morisawa, M., Kaneko, K., Nakaoka, S., Kanehiro, F., Tsuji, T., and Kajita, S. (2009). Toward human-like walking pattern generator. In International Conference on Intelligent Robots and Systems, 2009. IROS 2009, 1071-1077. IEEE.

Kim, S., Kim, C., You, B., and Oh, S. (2009). Stable wholebody motion generation for humanoid robots to imitate human motions. In IEEE/RSJ International Conference on Intelligent Robots and Systems, 2009. IROS 2009, 2518-2524. IEEE.

Miura, K., Morisawa, M., Nakaoka, S., Kanehiro, F., Harada, K., Kaneko, K., and Kajita, S. (2009). Robot motion remix based on motion capture data towards human-like locomotion of humanoid robots. In 9th IEEE-RAS International Conference on Humanoid Robots, 2009. Humanoids 2009, 596-603. IEEE.

Montecillo-Puente, F.J., Sreenivasa, M.N., and Laumond, J.P. (2010). On real-time whole-body human to humanoid motion transfer. In ICINCO, 22-31.

Nakamura, Y., Hanafusa, H., and Yoshikawa, T. (1987). Task-priority based redundancy control of robot manipulators. The International Journal of Robotics Research, $6(2), 3-15$.

Nakaoka, S., Nakazawa, A., Yokoi, K., Hirukawa, H., and Ikeuchi, K. (2003). Generating whole body motions for a biped humanoid robot from captured human dances. In Robotics and Automation, 2003. Proceedings. ICRA'03. IEEE International Conference on, volume 3, 39053910. IEEE.

Ramos, O.E., Saab, L., Hak, S., and Mansard, N. (2011). Dynamic motion capture and edition using a stack of tasks. In Humanoids, 224-230.

Schaal, S. (1999). Is imitation learning the route to humanoid robots? Trends in Cognitive Sciences, 3(6), 233-242.

Schaal, S., Ijspeert, A., and Billard, A. (2003). Computational approaches to motor learning by imitation. Philosophical Transactions of the Royal Society B: Biological Sciences, 358(1431), 537-547.

Suleiman, W., Monin, A., and Laumond, J. (2006). Synthesizing and modeling human locomotion using system identification. In Intelligent Robots and Systems, 2006 IEEE/RSJ International Conference on, 19721977. IEEE.

Yamane, K. and Hodgins, J. (2010). Control-aware mapping of human motion data with stepping for humanoid robots. In 2010 IEEE/RSJ International Conference on Intelligent Robots and Systems (IROS), 726-733. IEEE. 